\title{
Cardiovascular magnetic resonance
} (_) CrossMark evaluation of left ventricular peak filling rate using steady-state free precession and phase contrast sequences

\author{
Shotaro Komi ${ }^{1}$, Yusuke Inoue ${ }^{2^{*}}$, Hirofumi Hata ${ }^{1}$, Ai Nakajima ${ }^{1}$ and Hiroki Miyatake ${ }^{1}$
}

\begin{abstract}
Background: We investigated a practical method to measure peak filling rate (PFR) as an indicator of diastolic function of the left ventricle. Ten adult volunteers underwent cine MR imaging using steady-state free precession (SSFP) and phase contrast (PC) sequences to measure PFR. Two PC image sets were acquired at the mitral valve orifice, and PFR was determined from the set with high true temporal resolution (temporal PC method) or with high spatial resolution (spatial PC method). SSFP images covering the left ventricle were acquired, and a time-volume curve was generated around the peak filling phase. PFR was determined using parabolic curve fitting on the first-derivative curve of the LV time-volume curve.
\end{abstract}

Findings: PFR values estimated by the PC methods correlated well with those estimated by the SSFP method, despite apparent underestimation. The underestimation was smaller for the temporal PC method (12\%) than for the spatial PC method (28\%). Intra- and inter-observer repeatabilities were better for the PC methods than for the SSFP method.

Conclusions: PFR measurement by PC imaging with high true temporal resolution is convenient and offers excellent repeatability and acceptable accuracy, indicating suitability for clinical use.

Keywords: Cardiovascular magnetic resonance, Diastolic function, Peak filling rate, Phase contrast, Steady-state free precession

\section{Background}

Diastolic function of the left ventricle (LV) is often impaired before development of systolic dysfunction (Mandinov et al. 2000), and is commonly evaluated by echocardiography (Yamada and Klein 2010; Kasner et al. 2007; Oh et al. 2006). Cine cardiovascular magnetic resonance (CMR) imaging using a steady-state free precession (SSFP) sequence has been established as a reliable method for the measurement of LV volume and systolic function (Ichikawa et al. 2003; Attili et al. 2010; Finn et al.

\footnotetext{
${ }^{*}$ Correspondence: inoueys34@gmail.com

${ }^{2}$ Department of Diagnostic Radiology, Kitasato University School of Medicine, 1-15-1 Kitasato, Minami-ku, Sagamihara, Kanagawa 252-0374, Japan

Full list of author information is available at the end of the article
}

2006). Although the peak filling rate (PFR) can be also calculated as an indicator of diastolic function, the image analysis takes a long time because of the need for manual demarcation of the LV cavity on many slices, preventing widespread use of this method (Leong et al. 2010). Phase contrast (PC) cine CMR imaging can provide PFR values by measurement of mitral flow volumes (Rubinshtein et al. 2009; Beeres et al. 2008; Ashrafpoor et al. 2015). Manual tracing of the contour is required only on one slice at peak filling, and the convenience may make this method suitable for clinical use.

In the present study, we measured PFR in adult volunteers using SSFP and PC sequences to validate PFR measurement by PC imaging regarding that by SSFP imaging as a standard. High temporal resolution is essential for 
SSFP CMR assessment of PFR (Miller et al. 2002), which is assumed to hold true for PC CMR assessment. However, higher temporal resolution requires lower spatial resolution to avoid prolongation of scan time. We compared the effects of temporal resolution and spatial resolution on PFR measurement. In addition, we compared intra- and inter-observer variabilities between SSFP and PC methods. The aim of this study was to determine a practical CMR method to assess PFR.

\section{Methods}

\section{Subjects}

The study subjects comprised ten adult volunteers (seven males and three females) with no history of cardiac or chronic diseases and no contraindications to CMR imaging. The age was $30.5 \pm 6.2$ (mean $\pm \mathrm{SD}$ ) years, height was $169.5 \pm 10.5 \mathrm{~cm}$, and weight was $60.2 \pm 12.8 \mathrm{~kg}$. The heart rate, systolic blood pressure, and diastolic blood pressure were $69.1 \pm 6.6 \mathrm{bpm}, 125.4 \pm 15.3$, and $70.9 \pm 10.6 \mathrm{mmHg}$, respectively. The Institutional Review Board for Observation and Epidemiological Study, Kitasato University Medical Ethics Organization approved the study (KMEO B13-89), and all participants provided written informed consent.

\section{Imaging procedures}

All CMR studies were performed on a $1.5 \mathrm{~T}$ clinical scanner (Signa HDxt; GE Healthcare, Milwaukee, WI, USA) with an eight-channel phased-array coil. All images were obtained during breath holding at expiration. After localizing scans, vertical and horizontal long-axis images were obtained using an SSFP cine sequence.

On end-systolic images of both long-axis directions, one slice was planned for PC cine imaging at the position of the mitral valve orifice and in parallel to it. Two image sets, one with high true temporal resolution (temporal $\mathrm{PC}$ ) and one with high spatial resolution (spatial PC), were acquired using different views per segment (VPS) and different acquisition matrix sizes (Table 1). The reconstruction matrix $(256 \times 256)$ and reconstruction pixel size $(1.2 \times 1.2 \mathrm{~mm})$ were identical between both sets. Images at 64 cardiac phases were reconstructed

Table 1 Imaging parameters for PC cine MRI

\begin{tabular}{lll}
\hline Parameters & Temporal PC & Spatial PC \\
\hline TR (ms) & $5.4-5.5$ & $6.3-6.4$ \\
TE $(\mathrm{ms})$ & $3.6-3.7$ & $3.8-3.9$ \\
VPS & 4 & 8 \\
Acquisition matrix & $96 \times 96$ & $256 \times 192$ \\
Acquisition pixel size $(\mathrm{mm})$ & $3.1 \times 3.1$ & $1.2 \times 1.6$ \\
True temporal resolution $(\mathrm{ms})$ & $43.2-44.0$ & $100.8-102.4$ \\
\hline
\end{tabular}

using retrospective gating. Although the apparent temporal resolution, defined as the interval between adjacent reconstructed phases, was identical between the two sets and ranged from 13.8 to $17.0 \mathrm{~ms}$, depending on the heart rate, true temporal resolution, defined as TR $\times$ VPS $\times 2$ in PC cine imaging (Foo et al. 1995), was better for temporal PC imaging. One-dimensional velocity encoding was applied perpendicularly to the slice plane, and the velocity sensitivity threshold was set at $150 \mathrm{~cm} / \mathrm{s}$. Other scan parameters were as follows: flip angle, $20^{\circ}$; number of excitations (NEX), 1; FOV, $300 \times 300 \mathrm{~mm}$; slice thickness, $5 \mathrm{~mm}$; acquisition time, $14 \mathrm{~s}$ at a heart rate of $60 \mathrm{bpm}$. A parallel imaging technique [array spatial sensitivity encoding technique (ASSET)] was used with a reduction factor of 2 .

Contiguous short-axis images covering the entire LV were planned for SSFP cine imaging on end-diastolic SSFP images of both long-axis directions. Images at 32 cardiac phases were reconstructed using retrospective gating. The scan parameters were as follows: TR, $4.0 \mathrm{~ms}$; TE, $1.7 \mathrm{~ms}$; flip angle, 50 ; NEX, 0.5; FOV, $340 \times 340 \mathrm{~mm}$; slice thickness, $8 \mathrm{~mm}$; slice gap, $0 \mathrm{~mm}$; acquisition matrix, $224 \times 224$; VPS, 10. True temporal resolution, defined as TR $\times$ VPS in SSFP cine imaging, ranged from 39 to $40 \mathrm{~ms}$, and apparent temporal resolution varied from 27.6 to $34.1 \mathrm{~ms}$, depending on the heart rate. ASSET was used with a reduction factor of 2 , and the acquisition time was $9 \mathrm{~s} /$ slice at a heart rate of $60 \mathrm{bpm}$.

\section{Determination of PFR by PC imaging}

Dedicated software, $\mathrm{Cvi}^{42}$ (Circle Cardiovascular Imaging Inc., Calgary, $\mathrm{AB}$, Canada), was used for image analysis. On the phase images of PC imaging, an operator visually determined the peak filling phase during early filling. The region corresponding to the LV inflow through the mitral valve orifice was manually demarcated on the phase image at peak filling, referring to the magnitude image of PC imaging (Fig. 1). On phase images, the positivity or negativity of a

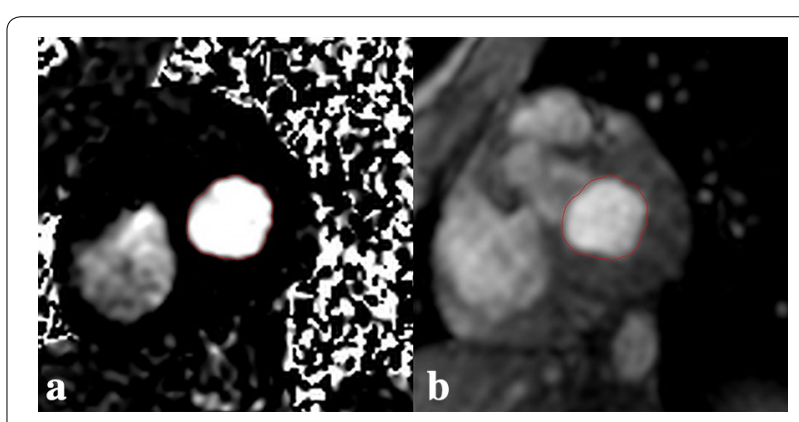

Fig. 1 Examples of the phase (a) and magnitude (b) images of PC cine imaging. Red lines indicate the contour of mitral flow traced manually 
pixel value represents the flow direction at the pixel. When the mitral flow produced positive pixel values, the operator set the upper and lower limits of the display window at 400 and 0 , respectively (i.e., window width, 400; window level, 200), in manual demarcation. In the case of negative values, the upper and lower limits of the display window were set at 0 and -400 , respectively (window width, 400; window level, -200$)$, and the gray scale was reversed. The mitral flow volume $(\mathrm{mL} / \mathrm{s})$ was estimated as the area multiplied by the absolute value of the mean signal intensity representing mean velocity. Considering possible inaccuracy of the visual determination of peak filling, the flow volume was also estimated at one cardiac phase each immediately before and immediately after the visual peak filling phase. PFR was defined as the maximum value among the flow volumes estimated at the three consecutive phases and was calculated from temporal PC and spatial PC images independently. Peak filling time was also recorded.

\section{Determination of PFR by SSFP imaging}

The peak filling phase during early filling was determined visually on the temporal PC images, and LV volumes were calculated using $\mathrm{cvi}^{42}$ from SSFP images obtained at five or six consecutive phases centered at the visual peak filling phase. Images were reconstructed at 32 and 64 cardiac phases in SSFP and PC images, respectively, and ideally the timing was identical between the $n$ phase ( $\mathrm{n}=$ natural number) in SSFP imaging and the $(2 \mathrm{n}-1)$ phase in PC imaging. When the peak filling appeared at the odd-numbered phase $(2 \mathrm{n}-1)$ in temporal PC imaging, five phases $(\mathrm{n}-2, \mathrm{n}-1, \mathrm{n}, \mathrm{n}+1, \mathrm{n}+2)$ were used for the analysis of SSFP images. When the peak filling appeared at the even-numbered phase (2n) in temporal PC imaging, the peak corresponded to the center between the $\mathrm{n}$ and $(\mathrm{n}+1)$ phases in SSFP imaging and six phases $(\mathrm{n}-2, \mathrm{n}-1, \mathrm{n}, \mathrm{n}+1, \mathrm{n}+2, \mathrm{n}+3)$ were used. At the phases for analysis, endocardial contours were traced manually on all short-axis images encompassing the LV cavity, and the LV volume was calculated. Papillary muscles were assigned to the LV cavity. On the basal slice including the LV outflow tract, the outflow tract and LV were divided by a straight line. The operator manually specified the basal end and apex of the LV on the vertical and horizontal long-axis views at each phase (Fig. 2), and the short-axis slices encompassing the LV were selected automatically. The partial volume effect inevitably caused mixing of the left atrium and LV within a pixel in the basal slice, and the software allowed separating the left atrium and LV within the basal slice through this processing on the long-axis images.

The first-derivative curve of the LV time-volume curve was generated by plotting the change rates of the LV

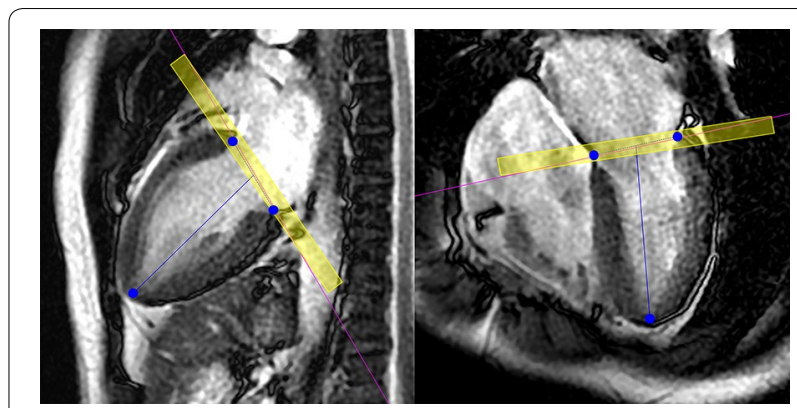

Fig. 2 Use of the long-axis views for LV volumetry from SSFP imaging. The operator manually specified the basal end and apex of the left ventricle (blue dots) on the vertical (left) and horizontal (right) long-axis views. The basal slice contained the left atrium and LV even within a pixel, and the processing using the long-axis images aided extraction of the true LV volume. The yellow box, representing the volume of the basal slice, was added for explanation to the actual display of the $\mathrm{Cvi}^{42}$ software

volume between two consecutive phases $(\mathrm{mL} / \mathrm{s})$ against time, and a parabolic curve was fitted to the first-derivative curve to preserve temporal resolution while reducing the effect of erroneous variation related to observerdependent contour demarcation. PFR and peak filling time were determined from the peak of the parabolic curve. Additionally, the end-diastolic volume (EDV), end-systolic volume (ESV), EF, and LV mass at end diastole were calculated from SSFP images. EDV, ESV, and mass were indexed to body surface area (BSA).

\section{Data analysis}

The relationship between PFRs estimated by two methods was assessed by linear regression analysis and Bland-Altman analysis. To evaluate intra- and interobserver variabilities in estimating PFR, one observer (S.K., 7 years of experience in CMR imaging) repeated the analysis with an interval of at least 2 weeks, and another observer (A.N., 4 years of experience in CMR imaging) analyzed the data independently. The intraand inter-observer variabilities were assessed by BlandAltman analysis.

\section{Statistical analysis}

Data are expressed as mean $\pm \mathrm{SD}$. Linear regression analysis was performed by the least squares method. Peak filling times obtained by three methods were compared using one-way analysis of variance. A $P$ value $<0.05$ was deemed statistically significant. The intraclass correlation coefficient (ICC) was calculated to evaluate inter- and intra-observer repeatabilities. The Statistical Package for Social Sciences (SPSS) version 21.0 for Windows (SPSS Inc., Chicago, IL, USA) was used. 


\section{Results}

Complete sets of SSFP and PC cine images were acquired in all ten subjects. The heart rate was $62.1 \pm 5.2 \mathrm{bpm}$ upon examination. EDV, ESV, EF, and mass were $149.5 \pm 25.9 \mathrm{~mL}$ (range 101.4-176.7 mL), $65.1 \pm 14.1 \mathrm{~mL}$ (range 40.2-81.1 mL), $56.7 \pm 3.1 \%$ (range 52.0-62.7 \%), and $94.9 \pm 25.6 \mathrm{~g}$ (range 52.1-129.2 g), respectively. EDV/BSA, ESV/BSA, and mass/BSA were $88.8 \pm 8.0 \mathrm{~mL} /$ $\mathrm{m}^{2}$ (range $76.8-99.8 \mathrm{~mL} / \mathrm{m}^{2}$ ), $38.5 \pm 5.2 \mathrm{~mL} / \mathrm{m}^{2}$ (range $29.3-46.5 \mathrm{~mL} / \mathrm{m}^{2}$ ), and $55.7 \pm 9.4 \mathrm{~g} / \mathrm{m}^{2}$ (range $39.4-$ $\left.68.2 \mathrm{~g} / \mathrm{m}^{2}\right)$, respectively.

PFR was estimated as $467.3 \pm 64.5,413.1 \pm 65.8$, and $335.4 \pm 53.9 \mathrm{~mL} / \mathrm{s}$ by the SSFP, temporal PC, and spatial PC methods, respectively. PFR values estimated by the temporal PC $[\mathrm{r}=0.825,95 \%$ confidence interval (CI) 0.408-0.957, Fig. 3a] and spatial PC $(r=0.781$, 95 \% CI 0.299-0.946, Fig. 3b) methods correlated well with those estimated by the SSFP method. Mean PFR estimated by the temporal PC method was $12 \%$ smaller than that estimated by the SSFP method. The bias was $-54.3 \pm 38.5 \mathrm{~mL} / \mathrm{s}(95 \% \mathrm{CI}-81.3$ to $-26.7 \mathrm{~mL} / \mathrm{s})$, the lower limits of agreement was $-129.8 \mathrm{~mL}(95 \%$ CI -177.5 to $-82 \mathrm{~mL} / \mathrm{s})$, and the upper limits of agreement was $21.2 \mathrm{~mL} / \mathrm{s}(95 \% \mathrm{CI}-26.5$ to $69.0 \mathrm{~mL} / \mathrm{s}$ ) (Fig. $4 \mathrm{a})$. Mean PFR estimated by the spatial PC method was $28 \%$ smaller than that estimated by the SSFP method. The bias was $-132.0 \pm 40.4 \mathrm{~mL} / \mathrm{s}(95 \% \mathrm{CI}-160.9$ to $-103.1 \mathrm{~mL} / \mathrm{s})$, the lower limits of agreement was $-211.2 \mathrm{~mL} / \mathrm{s}$ (95\% CI -261.3 to $-161.1 \mathrm{~mL} / \mathrm{s}$ ), and the upper limits of agreement was $-52.7 \mathrm{~mL} / \mathrm{s}(95 \%$ CI -102.8 to $-2.6 \mathrm{~mL} / \mathrm{s}$ ) (Fig. $4 \mathrm{~b}$ ). Peak filling time was estimated as $457.5 \pm 31.9 \mathrm{~s}, 466.2 \pm 18.2 \mathrm{~s}$, and $475.6 \pm 23.9 \mathrm{~s}$ by the SSFP, temporal PC, and spatial PC methods, respectively, showing no significant difference $(P=0.294)$.

Intraobserver comparisons demonstrated that the biases were close to zero for all methods, indicating limited systematic errors (Fig. 5). However, the $95 \%$ CIs were definitely wider and ICCs smaller (Table 2) for the SSFP method than for the PC methods, indicating larger random errors for the SSFP method. Interobserver comparisons revealed that both systematic and random errors were larger for the SSFP method than for the PC methods (Fig. 6; Table 2).

\section{Discussion}

To estimate PFR using SSFP cine CMR imaging, the LV contour is demarcated on many slices at many cardiac phases, which takes a long time. Because even minor fluctuation of the LV volume may cause a large error in the change rate of the volume (Theisen et al. 2013), the observer should be particularly careful in the demarcation. The considerable burden on observers has limited its widespread use. In the present study, manual demarcation was performed only around the peak filling phase determined visually on PC images, which reduced the observer's burden.

$\mathrm{PC}$ cine CMR imaging at the mitral valve orifice allow to estimate PFR via measurement of the LV inflow over time (Rubinshtein et al. 2009; Beeres et al. 2008; Ashrafpoor et al. 2015). This estimation requires data acquisition of only one slice and image analysis of only one cardiac phase, offering substantial convenience. The present study demonstrated that PFR estimated by the PC method correlated well with that estimated by the SSFP method, despite apparent underestimation. Although
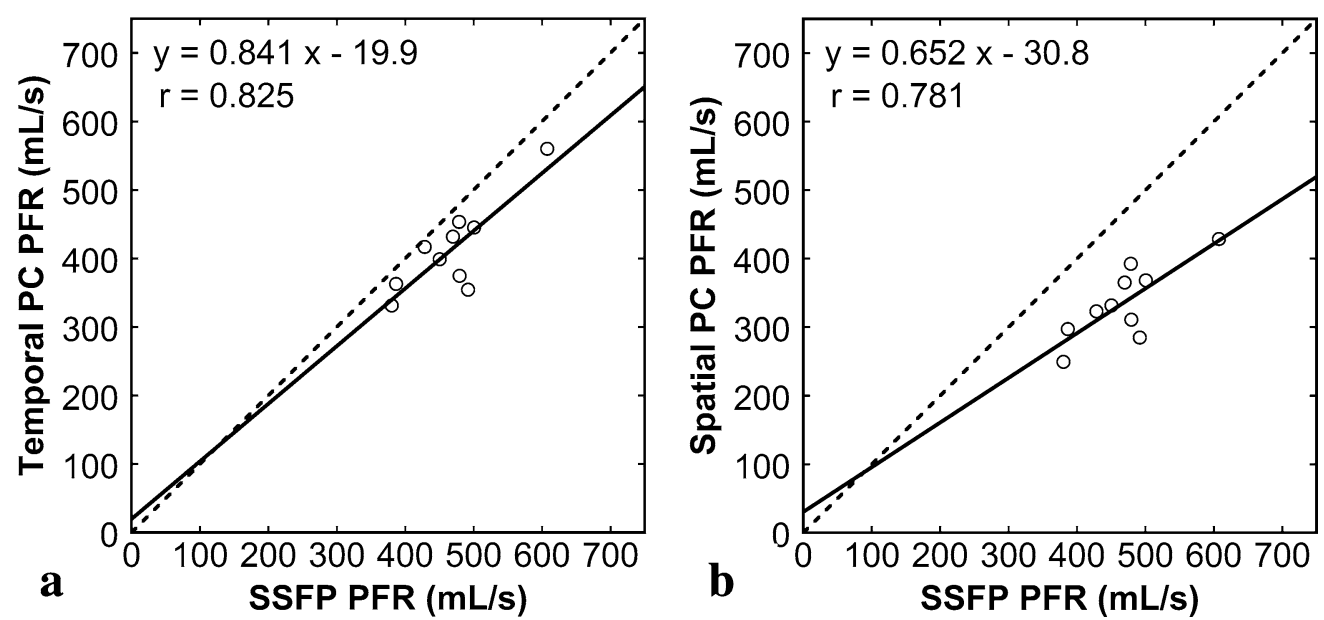

Fig. 3 Comparison of PFRs estimated by various methods. PFR values estimated by the temporal PC method (a) and spatial PC method (b) were plotted against those estimated by the SSFP method. The solid lines represent the regression lines, and the broken lines represent the line of identity 

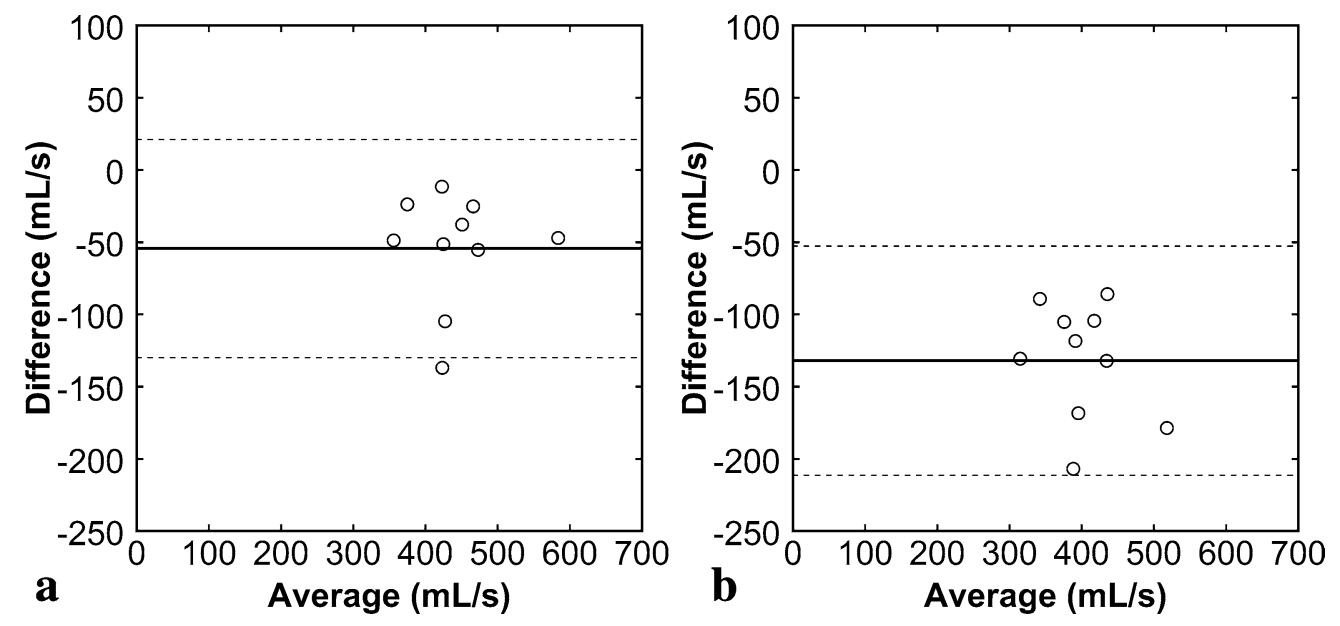

Fig. 4 Bland-Altman plots for PFRs estimated by various methods. The temporal PC method (a) and spatial PC method (b) were compared with the SSFP method. The difference between two methods was plotted against their average. The solid line represents the mean of the differences, and the broken lines represent the mean $\pm 1.96 \mathrm{SD}(95 \% \mathrm{Cl})$
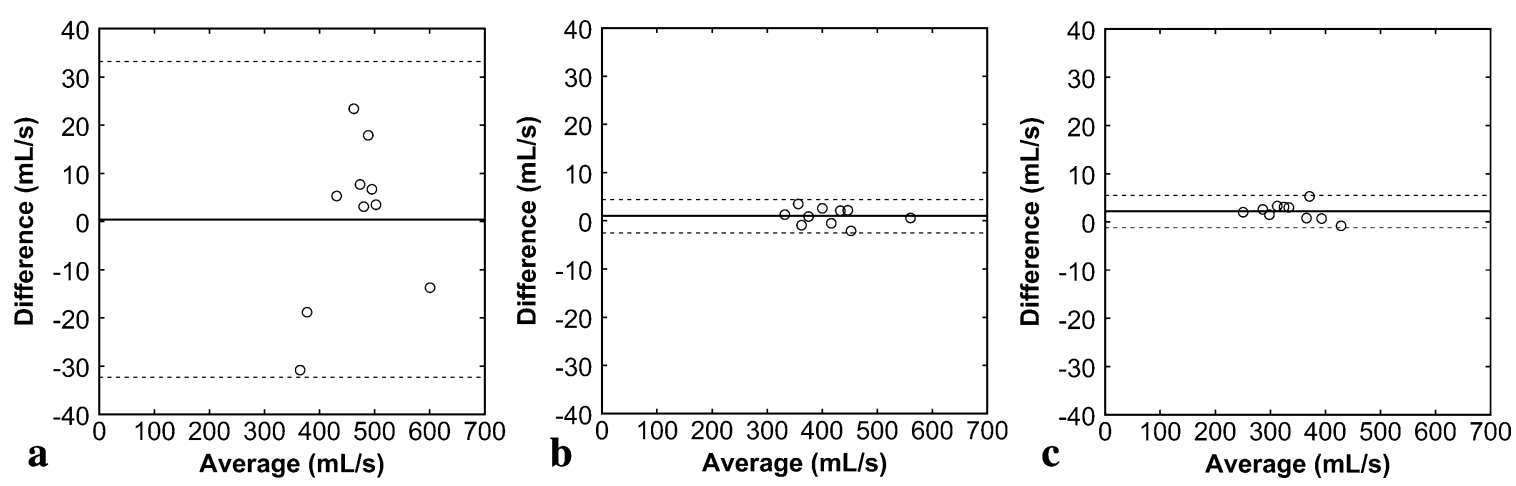

Fig. 5 Bland-Altman plots representing intraobserver variabilities (a SSFP method, b temporal PC method, c spatial PC method). The difference between the two estimates by an observer was plotted against their average. The solid line represents the mean of the differences, and the broken lines represent the mean $\pm 1.96 \mathrm{SD}(95 \% \mathrm{Cl})$

Table 2 ICC for intra- and inter-observer variability

\begin{tabular}{lll}
\hline Methods & Intraobserver & Interobserver \\
\hline SSFP & 0.972 & 0.871 \\
Temporal PC & 1.000 & 1.000 \\
Spatial PC & 0.999 & 0.999 \\
\hline
\end{tabular}

more sophisticated techniques such valve tracking may further improve accuracy (Brandts et al. 2011), the simple PFR measurement using PC cine imaging appears to be a practical and widely available method for evaluation of diastolic function with an acceptable burden on the patient and operator.

PFR estimates were compared between PC imaging with high true temporal resolution and that with high spatial resolution. PFR was systematically underestimated for both sets compared with PFR estimated by the SSFP method; however, the degree of underestimation was smaller using high true temporal resolution. It is suggested that true temporal resolution is more important than spatial resolution for measuring PFR by PC imaging. Improvement of true temporal resolution prolongs acquisition time and, consequently, breath-holding time. Reducing spatial resolution appears to be acceptable to prevent excessive prolongation of the acquisition time. In SSFP imaging, the influence of true temporal resolution is greater for PFR measurement than for EF measurement (Miller et al. 2002). It is suggested that PC imaging with low temporal resolution also underestimates PFR due to rapid changes in the filling rate. Apparent temporal resolution, defined as the interval between the adjacent reconstructed phases, was identical for the two sets of PC images with high true temporal resolution and high spatial resolution. In SSFP 

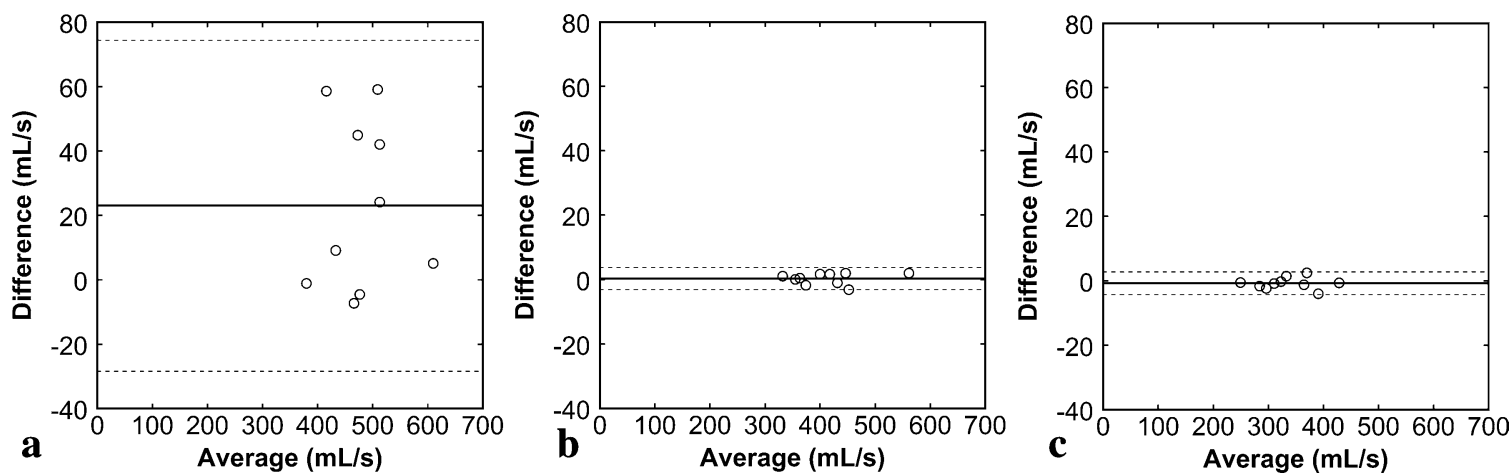

Fig. 6 Bland-Altman plots representing interobserver variabilities (a SSFP method, $\mathbf{b}$ temporal PC method, c spatial PC method). The difference between the two observers were plotted against their average. The solid line represents the mean of the differences, and the broken lines represent the mean $\pm 1.96 \mathrm{SD}(95 \% \mathrm{Cl})$

imaging, a reduction in true temporal resolution has been indicated to cause underestimation of EF even with high apparent temporal resolution (Inoue et al. 2005). The results of the present study have suggested that improvement of the apparent temporal resolution does not effectively compensate for low true temporal resolution in the measurement of PFR by PC imaging.

PFR values estimated from a given set of images may vary depending on observer-dependent demarcation of the LV contour. In the present study, better inter- and intraobserver repeatabilities in PFR measurement were demonstrated for the PC methods compared with the SSFP method. The SSFP method produced larger random errors in intraobserver comparison and larger systematic and random errors in interobserver comparison compared with the PC methods. The area of the demarcated LV region directly affects the estimation of PFR from SSFP images. By contrast, PFR measurement from PC images utilizes the total signal in the demarcated region and is less susceptible to observer-dependent contour demarcation than measurement from SSFP images due to low signal intensity near the contour. The excellent interand intraobserver repeatabilities support the clinical usefulness of PFR measurement by PC imaging.

In the present study, only a small number of adult volunteers were examined using CMR imaging alone. Because echocardiography is a current standard for the evaluation of diastolic function, comparison of the CMR method with echocardiographic method in a large number of patients with LV dysfunction should be conducted in the future.

\section{Conclusion}

We investigated methods of measuring PFR using CMR imaging. PFR values estimated by PC imaging correlated well with those estimated by SSFP imaging, despite systematic underestimation. In the measurement of PFR by PC imaging, true temporal resolution was more important than spatial resolution, and improvement in the apparent temporal resolution did not effectively compensate for low true temporal resolution. Inter- and intra-observer repeatabilities were better for the PC methods than for the SSFP methods. PFR measurement by PC imaging with high temporal resolution is convenient and offers excellent repeatability and acceptable accuracy, suggesting its suitability for clinical use as an adjunct to CMR assessment of systolic function.

\begin{abstract}
Abbreviations
Cl: confidence intervals; ICC: intraclass correlation coefficient; PC: phase contrast; PFR: peak filling rate; SSFP: steady-state free precession; VPS: views per segment.
\end{abstract}

\section{Authors' contributions}

SK carried out data acquisition, image analysis, statistical analysis, and drafting the manuscript. Yl conceived and designed the study, interpreted data, and performed critical revision of the manuscript. $\mathrm{HH}$ assisted the study design, data acquisition, image analysis, and drafting the manuscript. AN participated in data acquisition, carried out the image data analysis, and was involved in drafting the manuscript. HK carried out statistical analysis and was involved in revising the manuscript. All authors read and approved the final manuscript.

\section{Author details}

${ }^{1}$ Department of Radiology, Kitasato University Hospital, Sagamihara, Kanagawa, Japan. ${ }^{2}$ Department of Diagnostic Radiology, Kitasato University School of Medicine, 1-15-1 Kitasato, Minami-ku, Sagamihara, Kanagawa 252-0374, Japan.

\section{Competing interests}

The authors declare that they have no competing interests.

Received: 14 April 2016 Accepted: 20 July 2016

Published online: 25 July 2016

\section{References \\ Ashrafpoor G, Bollache E, Redheuil A, De Cesare A, Giron A, Defrance C, Azarine A, Perdrix L, Ladouceur M, Diebold B, Mousseaux E, Kachenoura N (2015)}


Age-specific changes in left ventricular diastolic function: a velocityencoded magnetic resonance imaging study. Eur Radiol 25:1077-1086. doi:10.1007/s00330-014-3488-z

Attili AK, Schuster A, Nagel E, Reiber JH, van der Geest RJ (2010) Quantification in cardiac MRl: advances in image acquisition and processing. Int J Cardiovasc Imaging 1:27-40. doi:10.1007/s10554-009-9571-x

Beeres SL, Lamb HJ, Roes SD, Holman ER, Kaandorp TA, Fibbe WE, de Roos A, van der Wall EE, Schalij MJ, Bax JJ, Atsma DE (2008) Effect of intramyocardial bone marrow cell injection on diastolic function in patients with chronic myocardial ischemia. J Magn Reson Imaging 27:992-997. doi:10.1002/jmri.21081

Brandts A, Bertini M, van Dijk EJ, Delgado V, Marsan NA, van der Geest RJ, Siebelink HM, de Roos A, Bax JJ, Westenberg JJ (2011) Left ventricular diastolic function assessment from three-dimensional three-directional velocity-encoded MRI with retrospective valve tracking. J Magn Reson Imaging 33:312-319. doi:10.1002/jmri.22424

Finn JP, Nael K, Deshpande V, Ratib O, Laub G (2006) Cardiac MR imaging: state of the technology. Radiology 241:338-354. doi:10.1148/ radiol.2412041866

Foo TK, Bernstein MA, Aisen AM, Hernandez RJ, Collick BD, Bernstein T (1995) Improved ejection fraction and flow velocity estimates with use of view sharing and uniform repetition time excitation with fast cardiac techniques. Radiology 195:471-478. doi:10.1148/radiology.195.2.7724769

Ichikawa Y, Sakuma H, Kitagawa K, Ishida N, Takeda K, Uemura S, Motoyasu M, Nakano T, Nozaki A (2003) Evaluation of left ventricular volumes and ejection fraction using fast steady-state cine MR imaging: comparison with left ventricular angiography. J Cardiovasc Magn Reson 5:333-342. doi:10.1081/JCMR-120019422

Inoue Y, Nomura Y, Nakaoka T, Watanabe M, Kiryu S, Okubo T, Ohtomo K (2005) Effect of temporal resolution on the estimation of left ventricular function by cardiac MR imaging. Magn Reson Imaging 23:641-645. doi:10.1016/j. mri.2005.04.004
Kasner M, Westermann D, Steendijk P, Gaub R, Wilkenshoff U, Weitmann K, Hoffmann W, Poller W, Schultheiss HP, Pauschinger M, Tschöpe C (2007) Utility of Doppler echocardiography and tissue Doppler imaging in the estimation of diastolic function in heart failure with normal ejection fraction: a comparative Doppler-conductance catheterization study. Circulation 116:637-647. doi:10.1161/CIRCULATIONAHA.106.661983

Leong DP, De Pasquale CG, Selvanayagam JB (2010) Heart failure with normal ejection fraction: the complementary roles of echocardiography and CMR imaging. JACC Cardiovasc Imaging 3:409-420. doi:10.1016/j. jcmg.2009.12.011

Mandinov L, Eberli FR, Seiler C, Hess OM (2000) Diastolic heart failure. Cardiovasc Res 45:813-825. doi:10.1016/S0008-6363(99)00399-5

Miller S, Simonetti OP, Carr J, Kramer U, Finn JP (2002) MR Imaging of the heart with cine true fast imaging with steady-state precession: influence of spatial and temporal resolutions on left ventricular functional parameters. Radiology 223:263-269. doi:10.1148/radiol.2231010235

Oh JK, Hatle L, Tajik AJ, Little WC (2006) Diastolic heart failure can be diagnosed by comprehensive two-dimensional and Doppler echocardiography. J Am Coll Cardiol 47:500-506. doi:10.1016/j.jacc.2005.09.032

Rubinshtein R, Glockner JF, Feng D, Araoz PA, Kirsch J, Syed IS, Oh JK (2009) Comparison of magnetic resonance imaging versus Doppler echocardiography for the evaluation of left ventricular diastolic function in patients with cardiac amyloidosis. Am J Cardiol 103:718-723. doi:10.1016/j. amjcard.2008.10.039

Theisen D, Sandner TA, Bamberg F, Bauner KU, Schwab F, Schwarz F, Arnoldi E, Reiser MF, Wintersperger BJ (2013) High-resolution cine MRI with TGRAPPA for fast assessment of left ventricular function at 3 Tesla. Eur J Radiol 82:219-224. doi:10.1016/j.ejrad.2012.12.019

Yamada H, Klein AL (2010) Diastology 2010: clinical approach to diastolic heart failure. J Echocardiogr 8:65-79. doi:10.1007/s12574-010-0055-8

\section{Submit your manuscript to a SpringerOpen ${ }^{\circ}$ journal and benefit from:}

- Convenient online submission

- Rigorous peer review

- Immediate publication on acceptance

- Open access: articles freely available online

- High visibility within the field

- Retaining the copyright to your article

Submit your next manuscript at $\boldsymbol{\nabla}$ springeropen.com 